\title{
INFLUENCE OF DEFECTS ON FERROELASTIC PHASE TRANSITION IN InI SINGLE CRYSTALS
}

\author{
M.I. Kolinko, I.V. KiTYK AND A.B. KozHLJUK \\ Physical Faculty, Lviv I. Franko University, Lomonosova 8a, Lviv, 290005, Ukraine
}

(Received March 30, 1993; revised version June 14, 1993)

\begin{abstract}
The influence of the defect subsystem on the behaviour of the structural incommensurate phase in InI single crystals was investigated. The correlation between the incommensurate parameter $\zeta$ and the piezooptical $\pi_{2222}$ tensor component was revealed.
\end{abstract}

PACS numbers: $77.80 . \mathrm{Bh}$

InI single crystals (space group $D_{2 h}^{17}$ ) are typical layered $A^{3} B^{7}$ compounds with a pronounced anisotropy of the crystalline structure and the corresponding optical properties [1-5]. At the same time, the recent revelation of the low temperature phase transitions in these crystals $[6,7]$ can present them as convenient model objects for the creation of phase transitions theory in high anisotropy media including those with the incommensurate phase. In the circumstances, it is very substantial that similar two-dimensional systems are most susceptible to the appearance of the translational symmetry perturbations of different kind, above all at the expense of appearance of defects, dislocations and vacancies. The last ones are in particular fashion exhibited in modulated superstructures [8].

The goal of this work is to describe the influence of the defect subsystem on phase transitions in InI single crystals, because the layering of structure allows one to control smoothly the corresponding parameters.

The initial samples were synthesized using reaction between the pure metallic indium and iodine in a hermetical quartz ampoule. After the band cleaning and distillation in the vacuum, single crystals have been grown by the Bridgman method [9]. Independently of crystal perfection all the cleaved monocrystalline specimens possessed mirror surfaces of high quality in ac-plane, which did not require additional polishing. The structural parameters were checked using the $\mathrm{X}$-ray DRON-05 diffractometer. The temperature measurements were performed in the range $4.2-500 \mathrm{~K}$ and were stabilized within $0.1-0.2 \mathrm{~K} \cdot \mathrm{min}^{-1}$. The Senarmon method $(\lambda=0.6328 \mu \mathrm{m})$ was used for measuring of the piezooptical constants.

Testing of samples quality was performed by the X-ray method. The broadening of X-ray satellite reflexes permits to select crystals of different quality. To obtain smooth change of the specimens quality, they were annealed in non-inertial 
media at $355 \mathrm{~K}$. For the quantitative identification of the samples quality, the dimensionless $\xi$ coefficient, which is equal to the ratio between the ion reflex diameters of the most perfect and less quality samples, was introduced. This is well justified for the compounds with the layered structures and the great anisotropy of chemical bond [10]. As a consequence, the samples with $\xi$ values in the range from 0.36 to 1.00 were selected.

Below $25 \mathrm{~K}$ in the X-ray sattelite reflexes of the more defective crystals one can distinctly notice a simultaneous coexisting of two structural phases. The first one is the stronger and belongs to the $D_{2 h}^{17}$ space group, and the second one is the weaker and belongs to the acentric $C_{2 v}^{9}$ phase.

Temperature dependencies of the incommensurable parameter determined from the X-ray satellites $(h, 0, l \pm \zeta)$ for samples with different defectiveness are displayed in Fig. 1. One can clearly see that the increase in annealing time of

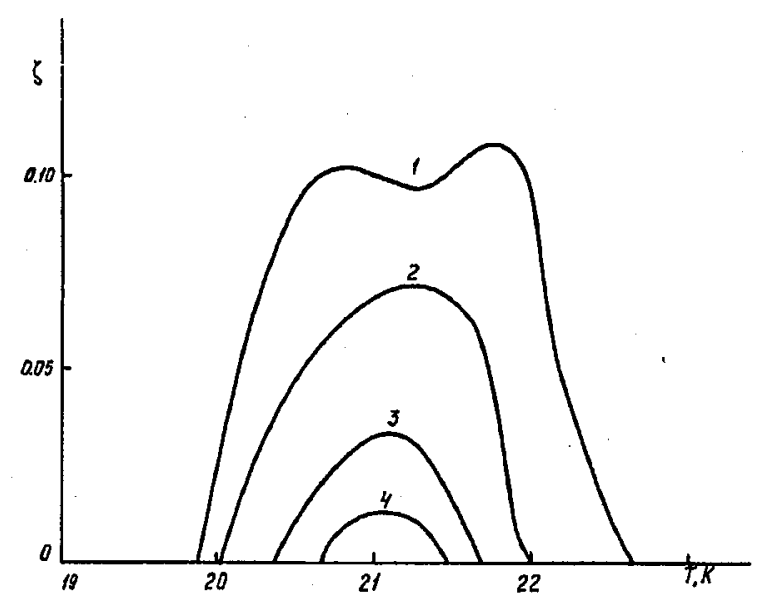

Fig. 1. The temperature dependencies of the incommensurable $\zeta$ parameter for the InI single crystals with the different defectiveness: $1-\xi=0.36,2-0.51,3-0.62$, $4-0.73$.

the samples (which corresponds to the increase in $\xi$ parameter) is accompanied with narrowing of the temperature range of the incommensurate phase existence. Begining from $\xi>0.75$, the incommensurability was lacking. Besides, it is essential that as the quality of samples decreases, the superstructure modulation increases. The temperature changes of the lattice parameters $(a, b, c)$ for the samples of the different content of defects in the vicinity of the phase transition are shown in Table. It was found that the $a$ and $b$ parameters are practically independent of the structural reorientation. The pronounced modulation exists simultaneously along the $c$ axis (i.e., along the weak cation-cation interlayer bonds $[11,12])$. The attempts to reveal the ferroelectric spontaneous polarization were unsuccessful, because within the Soyer-Tower method precision $\left(f=10 \mathrm{kHz}, E \leq 10^{6} \mathrm{~V} \cdot \mathrm{m}^{-1}\right.$, $\left.\Delta P_{\mathrm{s}} \approx 2 \times 10^{-4} \mu \mathrm{C} \cdot \mathrm{cm}^{-2}\right)$ the existence of the nonzero value $P_{\mathrm{s}}$ was not noticed. At the same time, the earlier performed dilatometric measurements $[6,7]$ showed 
TABLE

Temperature changes of the InI lattice parameters in the vicinity of the phase transitions for the samples of the different quality.

\begin{tabular}{l|c|c|c|c|c|c}
\hline \hline$T[\mathrm{~K}]$ & $a[\mathrm{~nm}]$ & $b[\mathrm{~nm}]$ & \multicolumn{5}{|c}{$c[\mathrm{~nm}]$} \\
\cline { 4 - 7 } & & & $\zeta=0.36$ & $\zeta=0.52$ & $\zeta=0.62$ & $\zeta=0.73$ \\
\hline 19.8 & $0.4752(3)$ & $1.2759(1)$ & $0.4908(0)$ & $0.4908(0)$ & $0.4908(0)$ & $0.4908(0)$ \\
20.0 & $0.4752(5)$ & $1.2759(3)$ & $0.5013(3)$ & $0.4909(2)$ & $0.4909(8)$ & $0.4909(8)$ \\
20.3 & $0.4752(8)$ & $1.2759(7)$ & $0.5317(4)$ & $0.5046(2)$ & $0.4910(8)$ & $0.4910(1)$ \\
20.6 & $0.4753(1)$ & $1.2760(0)$ & $0.5340(6)$ & $0.5134(3)$ & $0.5042(6)$ & $0.4924(3)$ \\
20.8 & $0.4753(2)$ & $1.2760(2)$ & $0.5502(2)$ & $0.5187(2)$ & $0.5101(4)$ & $0.5012(6)$ \\
21.0 & $0.4753(4)$ & $1.2760(4)$ & $0.5447(3)$ & $0.5239(3)$ & $0.5089(6)$ & $0.5014(5)$ \\
21.4 & $0.4753(5)$ & $1.2760(5)$ & $0.5459(4)$ & $0.5251(6)$ & $0.5013(7)$ & $0.4912(6)$ \\
21.8 & $0.4753(8)$ & $1.2760(8)$ & $0.5558(3)$ & $0.5201(7)$ & $0.4911(1)$ & $0.4911(8)$ \\
22.0 & $0.4753(9)$ & $1.2760(9)$ & $0.5558(8)$ & $0.5013(8)$ & $0.4911(5)$ & $0.4911(7)$ \\
22.3 & $0.4754(1)$ & $1.2761(1)$ & $0.5080(7)$ & $0.4911(8)$ & $0.4911(8)$ & $0.4911(9)$ \\
22.6 & $0.4754(2)$ & $1.2761(3)$ & $0.4912(3)$ & $0.4913(7)$ & $0.4912(6)$ & $0.4912(8)$
\end{tabular}

the occurrence of the spontaneous deformation in the temperature range 18-22 K, which allows one to classify unambiguously the above mentioned transition as the ferroelastic one.

Therefore the investigation of piezooptical $\pi_{2222}$ and $\pi_{3333}$ components behaviour in the vicinity of the incommensurate phase is a vital problem. From Fig. 2 one can see that the $\pi_{2222}$ component is essentialy sensitive to the defectiveness of samples near the phase transition. In previous works $[6,7]$ it was noted that the $\pi_{3333}$ constant is insensitive to such kinds of influence. The temperature hysteresis within the $5 \mathrm{~K}$ is clearly observed, which points to the first order feature of this phase transition. With the upgrading of the samples quality there appear, on the one hand, the diminution of the $\pi_{2222}$ maximal value near phase transition, and, on the other hand, the decrease in the temperature range of incommensurate phase existence. Therefore, a correlation between the incommensurability parameters and corresponding optical susceptibilities exists clearly.

It is necessary to note that in the isostructural and isoelectronic $\mathrm{InBr}$ the analogous phenomena were absent.

To explain the observed changes of the incommensurate phase two models were proposed, particularly that of "catching" of the incommensurable region with the structural defects [13] and that of ordering of the defects or the impurities in the field of the modulated structure $[14,15]$. In the first one the most probable mechanism of the incommensurate phase appearance is concerned with the elastic interactions and the corresponding reorientation of the local dipole momenta in the twinning regions, which is obviously forbidden for the layered compounds. The obtained experimental results can be explained coming out from the second theoretically more complete model. In line with the last, if time of the temperature 


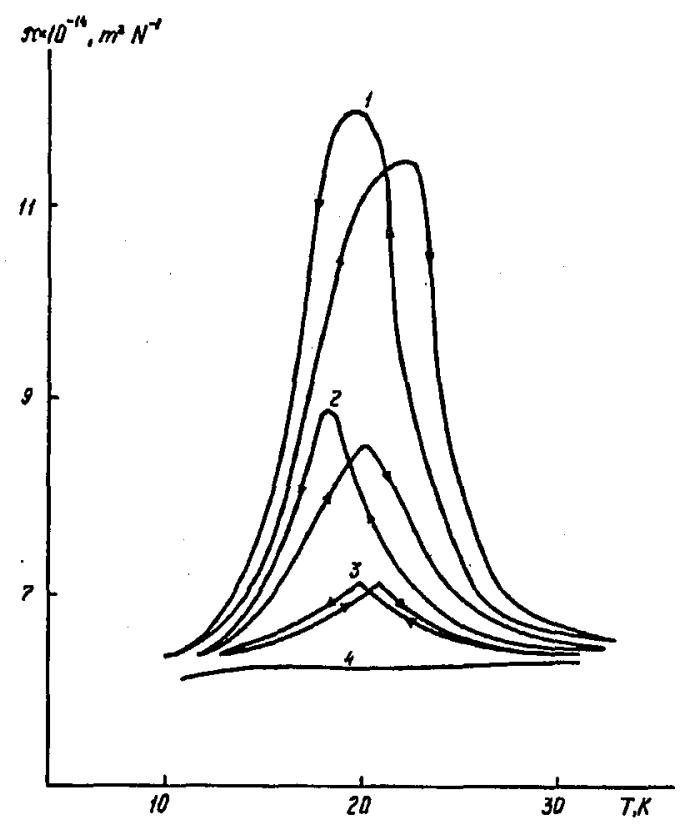

Fig. 2. The temperature hysteresis of the piezooptical $\pi_{2222}$ constants for InI single crystals of the different sample perfection: $1-\xi=0.36,2-0.51,3-0.62,4-0.73$.

stabilization is longer than of the relaxation of the migration defects, then the crystal gives birth to the wave with the changing density of defects distribution and with the period which is equal to that of the superstructure at this temperature. As a result "the frozen" wave is a function of the presence of defects in samples, which was clearly observed in the obtained results. Perhaps in this case, in consequence by interlayer interactions the membrane type vibrations (bending wave) become very essential.

\section{References}

[1] N. Ohno, M. Fujita, Y. Nakai, K. Nakamura, Solid State Commun. 28, 137 (1978).

[2] I.V. Blonskij, A.S. Krochuk, T.L. Stetsishin, A.V. Franiv, Sov. Phys.-Solid State 28, 1764 (1986).

[3] F. Jin, T. Itoh, T. Goto, J. Phys. Soc. Jpn. 58, 2586 (1989).

[4] A.S. Krochuk, I.V. Kityk, M.I. Kolinko, A.V. Franiv, Zh. Prikl. Spektrosk. 55, 142 (1991).

[5] A.S. Krochuk, I.V. Kityk, M.I. Kolinko, A.V. Franiv, Izv. Akad. Nauk SSSR, Neorg. Mater. 28, 619 (1992).

[6] M.I. Kolinko, I.V. Kityk, A.S. Krochuk, Fiz. Nizk. Temp. 18, 901 (1992).

[7] I.V. Kityk, M.I. Kolinko, A.V. Franiv, Ferroelectrics 130, 347 (1992). 
[8] G.A. Smolenskij, V.A. Bokov, V.A. Isupov, N.I. Krajnik, R.E. Pasynkov, I.I. Sokolov, I.K. Jushin, The Physics of Ferroelectric Phenomena, Nauka, Leningrad 1985.

[9] T.I. Dardwojt, E.G. Morozov, I.I. Vasilevskaja, Nauch. Trudy Giredmet 29, 25 (1970).

[10] K. Celly, X-Ray Crystallogr. 10, 176 (1987).

[11] M.I. Kolinko, Ukr. Fiz. Zh. 37, 117 (1992).

[12] A.S. Krochuk, I.V. Kityk, M.I. Kolinko, Fiz. Tekh. Poluprovodn. 26, 1028 (1992).

[13] H.-G. Unruh, J. Phys. C, Solid State Phys. 26, 3245 (1983).

[14] H.-G. Unruh, Ferroelectrics 53, 319 (1984).

[15] P. Lederer, G. Montambaus, J.P. Lamet, M. Chauwin, J. Phys. Lett. 45, 627 (1984). 